Original Article

\title{
HYDROALCOHOLIC EXTRACTION OF SHOOT CULTURES FROM NOTHAPODYTE NIMMONIANA AND ITS ANTI-PROLIFERATIVE ANALYSIS
}

\author{
ARUN SHARMA1, ANKITA RAJATA², UDAYABANU MALAIRAMAN ${ }^{*}$, HEMANT SOOD*
}

${ }^{1}$ Department of Pharmacy, Jaypee University of Information Technology, Waknaghat, Himachal Pradesh, India 173234, ${ }^{2}$ Department of biotechnology and bioinformatics, Jaypee University of Information Technology, Waknaghat, Himachal Pradesh, India 173234

Email: hemant.sood@juit.ac.in

Received: 07 Mar 2018 Revised and Accepted: 20 Apr 2018

ABSTRACT

Objective: The important restraints in plant-derived medicine are the convenience of active composites which depends on diverse topographical situations, active compound build-up pattern, environmental circumstances and genetic makeup of the plant. The presented work was aimed to describe an efficient technique for fast propagation of Nothapodytes nimmoniana which is an imperative source of Camptothecin.

Methods: Multiple shoots were successfully regenerated from the explants N. nimmoniana using Murashige and Skoog (MS) medium accompanied with IBA+KN+GA3 (1:3:2) mg/l. the hydro-alcoholic extract was obtained by soxhlet extraction for field grown roots (fgRE), shoots (fgSE) and in vitro plantlets (ivPL) and further subjected to phytochemicals analysis. HPLC analysis was performed to determine the percentage of camptothecin (CAM). Anti-proliferative studies were carried out followed by Acridine orange/Ethidium bromide staining. Extracts were also estimated for reactive nitrogen species (RNS) and reactive oxygen species (ROS).

Results: Preliminary qualitative chemical analysis of the extracts displayed the presence of alkaloids, carbohydrates, steroids, saponins, terpenoids and phenolics compounds. HPLC analysis revealed the presence of CAM in all the extracts. ivPL exhibited lowest anti-proliferative activity in contrast to other extract and percentage of ROS and RNS was also less. Although ivPL did not exhibited prominent anti-tumour effect, but in vitro accumulation of CAM in 2-months old shoots provides an alternative resource to $5 \mathrm{y}$-old field grown plants.

Conclusion: This work delivers a potential to lead to further increase the potency of CAM in in vitro shoots of two months old as an alternative to the field grown plants for treating cancers.

Keywords: Nothapodytes nimmoniana, Phytochemical, Plant tissue Culture, Anti-cancer activity, Camptothecin, HPLC

(c) 2018 The Authors. Published by Innovare Academic Sciences Pvt Ltd. This is an open access article under the CC BY license (http://creativecommons.org/licenses/by/4.0/) DOI: http://dx.doi.org/10.22159/ijpps.2018v10i6.25726

\section{INTRODUCTION}

Cancer is one of the major human ailments due to its manifestation and high mortality percentage that causes considerable suffering and economic loss worldwide at the extremely alarming rate. Therefore, further research is required for the advancement of safer drugs to prevent and treat human cancers. Almost $60 \%$ of drugs presently used for cancer treatment have been isolated from natural products and the plant kingdom has been the most significant source. One such medicinally vital plant is Nothapodytes nimmoniana Graham (common name-"Stinking tree, Ghanera") belonging to the family Icacinaceae [1] $\mathrm{N}$. nimmoniana is a well-known source of potent antitumor quinoline alkaloid; Camptothecin (CAM) [2], that has been well reported to cure cancers for instance breast, lungs and uterine cervical cancers [3-5]. CAM and its structural analogy have appeared as one of the most efficient anticancer moieties. Numerous CAM derivatives (Topotecan and Irinotecan) have been approved by the US-FDA and are already in the market as anticancer drugs [6, 7], whereas other derivatives have entered clinical trials against different types of cancer [8].

Thus, there is an incredible necessity to look upon that different measure which will escalate the source of CAM or we should examine the protocol for the mass proliferation through tissue culture technique in ordered to boost the scale production of CAM. Though some reports are there, regarding in vitro regeneration of $N$. nimmoniana by employing diverse explants, but none of them has recognized procedures related to the biochemical constancy of mature regenerants. There may be soma-clonal disparity and genetic instability in regenerating part and this may be attributive to the increased stress of in vitro culture conditions [9]. Therefore, assessment of clonal constancy biosynthesized medicinal compound analysis and advancement in plant development systems of the in vitro grown plants of $N$. nimmoniana will be of great implication.
Keeping all these facts in mind, the current examination was undertaken to create an effective procedure for the speedy clonal proliferation of $N$. nimmoniana through shoots germinated from somatic embryos [10]. In addition to that, biochemical estimation was done and further the studies were conducted to estimate antiproliferative and anti-oxidative fidelity.

\section{MATERIALS AND METHODS}

\section{Material}

Unless specified all the reagents and chemicals were procured from Sigma Aldrich.

\section{Selection of plant material}

N. nimmoniana was obtained from GKVK, University of Agricultural Sciences Bangalore. The plant was implanted and developed within the poly-house for five to six years at experimental premises of Department of Biotechnology and Bioinformatics, Jaypee University of Information Technology (JUIT), Waknaghat, India.

\section{Preparation of media and culture conditions}

MS (Murashige and Skoog) [11] medium accompanied with distinct concentrations and mixtures of growth hormones, for instance, TDZ $(1.5 \mathrm{mg} / \mathrm{l})$ and IBA $(1 \mathrm{mg} / \mathrm{l})$ with sucrose $3 \%(\mathrm{w} / \mathrm{v})$ have been used for somatic embryo formation and then conditions have been optimized to form somatic seed. Finally, the best germination of somatic seeds was achieved in M S medium supplemented with IBA+KN+GA3 (1:3:2) mg/l as reported in Ankita et al., 2017. So, the germinated shoots were used as the starting material for sub culturing on the same medium to develop in vitro grown plants. The axenic cultures were incubated for $16 \mathrm{~h}$ light cycle $\left(25 \pm 2{ }^{\circ} \mathrm{C}\right)$ in plant tissue culture (PTC) compartment. 


\section{Preparation of crude extract}

The young shoots or stems, roots of $N$. nimmoniana (25g) were washed under running tap water, shade dried, further grounded and subjected to hydroalcoholic (70:30; water: methanol) soxhlet extraction for $72 \mathrm{~h}$. The residue was discarded and the solvent was concentrated under reduced pressure using a rotary evaporator to remove the solvent and lyophilized afterwards.

\section{Preliminary phytochemical investigation}

The preliminary phytochemical screening was performed by employing previously defined procedures and it was ensured that powdered material was entirely dried and freed from traces of preceding solvents. Afterwards, the extracts were subjected to qualitative chemical tests [12].

\section{Phytochemical Analysis through HPLC}

Assessment of CAM was achieved through high-pressure liquid chromatography (HPLC) [13,14]. The investigation was done by using C18 symmetry (250MM $4.6 \mathrm{~mm}, 50 \mu$ ). The solvent system was acquired by mixing different solutions; $600 \mathrm{ml}$ (acetic acid- $10 \mathrm{ml}$ into distilled water- $600 \mathrm{ml}$ ) and $400 \mathrm{ml}$ (acetonitrile-200 ml+methanol$200 \mathrm{ml}$ ), followed by filtering the subsequent Solvents and degassing. The flow rate was attuned to $1 \mathrm{ml} / \mathrm{min}$ and the peak was recorded at the wavelength of $360 \mathrm{~nm}$. HPLC examination of extracts produced chromatograms with a retention time of $7 \mathrm{~min}$ for CAM.

\section{Cell lines and culture}

MCF-7, HEK-293 and MDA-MB-231 were procured from NCCS, Pune. Cells were cultured in tissue culture flasks comprising Dulbecco's modified Eagle medium (DMEM) for HEK-293 and MCF-7 similarly L-15 (Leibovitz) for MDA-MB-231 enriched by 10\% fetal bovine serum and $1 \%$ antibiotics $(100 \mathrm{U} / \mathrm{ml}$ penicillin and $100 \mu \mathrm{g} / \mathrm{ml}$ streptomycin). HEk-293 and MCF-7 cells were incubated at $37{ }^{\circ} \mathrm{C}$ containing $5 \% \mathrm{CO}_{2}$. On attainment confluence, the cells were subcultured further.

\section{Cytotoxicity studies and determination of IC $_{50}$}

Anti-proliferative effect of different extracts (fgRE, fgSE, and ivPE) was investigated through MTT assay conferring to previously wellestablished technique with slight modification [15] on three different cell lines (HEK-293, MCF-7, and MDA-MB-231). Briefly, cells were incubated with fgRE, fgSE and ivPE for $24 \mathrm{~h}$ at varying concentration $(125,250,500$ and $750 \mu \mathrm{g} / \mathrm{ml})$. Afterwards, the supernatant was discarded, $20 \mu \mathrm{l} \mathrm{MTT}(5 \mathrm{mg} / \mathrm{ml})$ was added to each well and incubated at $37{ }^{\circ} \mathrm{C}$ for further $4 \mathrm{~h}$. MTT was reduced by metabolically active cell (succinate dehydrogenase enzyme) to form insoluble purple colored formazan. 100- $\mu \mathrm{L}$ of Dimethyl sulfoxide (DMSO) was further added to each well to dissolve the formazan crystal and kept for 15-20 min at room temperature. Absorbance's (A) were taken at $570 \mathrm{~nm}$ as test wavelength and $630 \mathrm{~nm}$ as reference wavelength to assess cell cytotoxicity by using microplate reader (Bio-Rad). Triplicate wells were analyzed for each condition and standard deviations were calculated.

The cell cytotoxicity was determined through following equation:

$$
\left.\% \text { cell cytotoxicity }=\frac{A b s \text { control }}{A b s t} \times 100 \text { (Eq. no- } 1\right)
$$

Plots of percentage cytotoxicity index verses concentration were drawn and IC 50 (extract concentration resulting in $50 \%$ cytotoxicity) was determined from the graph.

\section{Acridine orange (AO) and ethidium bromide (EtBr) staining}

Further, the cells (HEK-293, MCF-7, and MDA-MB-231 cells) were investigated for morphological changes through dual $\mathrm{AO} / \mathrm{EtBr}$ staining. Briefly, cells were cultured at a density of $2 \times 10^{5}$ cells/well in 6-well plate supplemented with growth medium $(1 \mathrm{ml})$, till the confluence reached $70-80 \%$. Cells were then treated with selected concentration (IC 50 value for MCF-7) of each extract (fgRE, fgSE, and ivPE) and incubated for $24 \mathrm{~h}$. Afterwards, cells were collected, subjected to washing through phosphate-buffered saline (PBS) followed by fixing ( $4 \%$ of paraformaldehyde) for $30 \mathrm{~min}$. The supernatant was removed, cells were treated with $1 \%$ triton $\times 100$ for $15 \mathrm{~min}$ and further subjected for washing (PBS). Cells were stained with; $5 \mu \mathrm{L}$ of $\mathrm{AO}(1 \mathrm{mg} / \mathrm{ml})$ and $5 \mu \mathrm{L}$ of $\operatorname{EtBr}(1 \mathrm{mg} / \mathrm{ml})$ for 5 min and then washed three times with PBS. The cells were investigated for resulting morphological changes through fluorescence microscope at $\times 200$ (Nikon Eclipse-80i, Japan).

\section{Reactive nitrogen species (RNS) quantification}

Nitric oxide fall under the category of active RNS and considered as a major hallmark to cause cellular damage and apoptosis. Accordingly, we evaluated the NO levels as an effect of different extract effect at an individual $\mathrm{IC}_{50}$ value through Griess reagent [16]. Cells were treated with fgRE, fgSE and ivPE for $24 \mathrm{~h}$ and after completion of incubation time period, $75 \mu \mathrm{l}$ of supernatant was procured and 150 $\mu \mathrm{l}$ Griess reagent was induced to each well. Absorbance was taken spectrophotometrically at $550 \mathrm{~nm}$ and level of NO was calculated through following equation:

$$
\text { Percentage NO level }=\frac{(A) \text { of Sample }}{(A) \text { Control }} \times 100 \text { (Eq. no-2) }
$$

\section{Reactive oxygen species (ROS) quantification}

To examine ROS formation as result of extract exposure, we quantified cellular $\mathrm{H}_{2} \mathrm{O}_{2}$ amount by employing readialy available kit for in vitro $\mathrm{H}_{2} \mathrm{O}_{2}$ cytotoxicity estimation (PerXOquant ${ }^{\mathrm{TM}}$ Quantitative peroxide assay kits) [16]. This assay specifically evaluates the $\mathrm{H}_{2} \mathrm{O}_{2}$ formation in solution by changing $\mathrm{Fe}^{2+}$ to $\mathrm{Fe}^{3+}$ at acidic $\mathrm{pH}$, results in purple color accordingly. Cells were incubated with fgRE, fgSE and ivPE for $24 \mathrm{~h}$ and then centrifuged at $1 \times 10^{4} \mathrm{~g}$ for $10 \mathrm{~min}$. Further, 50 $\mu \mathrm{l}$ of supernatant was removed and relocated in fresh flat bottom 96-well microplate comprising $200 \mu$ l of working reagent (standard manufacturer's protocol) and further the absorbance was recorded spectrophotometrically at $595 \mathrm{~nm}$. The concentration of $\mathrm{H}_{2} \mathrm{O}_{2}$ was determined from the standard curve $\left(\mathrm{H}_{2} \mathrm{O}_{2} \quad 0-100 \mu \mathrm{M}\right)$ and the percentage of $\mathrm{H}_{2} \mathrm{O}_{2}$ was calculated through following equation:

$$
\text { Percentage } \mathrm{H}_{2} \mathrm{O}_{2} \text { level }=\frac{(\text { A }) \text { of sample }}{(\text { A }) \text { of control }} \times 100 \text { (Eq. no-3) }
$$

\section{Statistics}

Statistical analysis was carried out through Graph Pad Prism version 5.0 for Windows (Graph Pad Software, San Diego, CA, USA). One-way ANOVA followed by Dunnett post hoc tests was used to calculate the statistical significance of data at ${ }^{* * *} \mathrm{p}<0.001,{ }^{* *} \mathrm{p}<0.01$ and ${ }^{*} \mathrm{p}<0.05$. Results are represented as mean \pm SEM.

\section{RESULTS AND DISCUSSION}

\section{In vitro shoot multiplication}

The germinated shoots from the somatic embryos of N. nimmoniana developed by Ankita et al. 2017, were used for sub-culturing on MS medium augmented with IBA+KN+GA3 (1:3:2) mg/l and incubated for $4 \mathrm{w}$ as mentioned before in the optimized culture conditions. The in vitro micro shoots developed into thick well-grown plantlets after one sub-culturing so two months old shoots were further used for all the experimentation work mentioned ahead (fig. 1).

\section{Preliminary qualitative investigation}

Characteristic phytochemical assessment exhibited the existence of alkaloids, carbohydrates, saponins, steroids, terpenoids and phenolics compounds in the all extracts (fgRE, fgSE and ivPE) of $N$. nimmoniana.

\section{Phytochemical analysis}

The quantitative investigation of the CAM content of distinct extracts, viz, fgRE, fgSE and ivPE was performed through HPLC and indicated that the amount of CAM was variable. The peak area was acquired at the wavelength of $360 \mathrm{~nm}$ and the chromatograms attained in our work was developed on the same line. Retention time for standard camptothecin was 2.8 min covering an area of 833.834 (fig. 2a). fgSR had a retention time of 2.797 and total CAM quantified was $0.87 \%$ (fig. 
2b). The amount of CAM quantified in fgSE was $0.17 \%$ with a retention time of 2.791 (fig. 2c). ivPE have very little CAM content $(0.03 \%)$ as shown in Fig.2d with a retention time of 2.805 min.

HPLC assessment revealed that more content of CAM was present in field grown root extract (fgRE) followed by field has grown shoot extract (fgSE). Least content of CAM was been measured in in vitro grown plantlets extract (ivPE).

\section{Cytotoxic evaluation}

MTT assay is a reliable technique that reveals the cell viability on the bases of mitochondrial dehydrogenase action, widely used to screen-out anti-proliferative agents. Fig.3 presented the plots of cytotoxicity (\%) versus concentrations of different extracts (fgRE, fgSE and ivPE). Almost every individual extract showed the antiproliferative effect on cancerous cell lines (MCF-7 and MD-MBA231 ) in the dose-dependent miner. Table 1 , represents the $\mathrm{IC}_{50}$ values, calculated from the graphs and the maximum antiproliferative effect was observed with fgRE with an $\mathrm{IC}_{50}$ value of 704 and $652 \mu \mathrm{g} / \mathrm{ml}$ for MCF-7 and MDA-MB-231 cell line. Higher concentration of CAM can be attributive to the potent anti-cancer activity of fgRE. Likewise, fgSE represented $\mathrm{IC}_{50}$ value of 1079.53 and $1025 \mu \mathrm{g} / \mathrm{ml}$ for MCF-7 and MDA-MB-231 cells. Whereas, ivPE exhibited an $\mathrm{IC}_{50}$ value of 2997.75 and $2850.75 \mu \mathrm{g} / \mathrm{ml}$ for MCF-7 and MDA-MB-231 cells. Although the performance of ivPE was not found to better than field grown root and shoot extract. But, it was interesting to note that the extract was only of 2-months grown a plant under laboratory conditions, in comparison to 5-years grown plant.

Effect of extracts on normal epithelial HEK-293 cell line and found to less toxic when compared on the bases of IC50 values (supporting Data S-1).

\section{Cell morphological evaluation through $\mathrm{AO} / \mathrm{EtBr}$}

Further to have better insight into the extract induced cell killing behaviour we performed A0/EtBr staining on HEK-293, MCF-7, and MDA-MB-231 cells. Corresponding $\mathrm{IC}_{50}$ values of the different extracts were selected for this experiment and the conclusion was drawn on the basis of mean fluorescence intensity for red color (EtBr-stained dead cells) by employing Image-J software (fig. 4).

Green fluorescence is the distinctive feature of healthy and live state of cells, whereas yellow stain signifies early apoptosis and reddish or orange stain represents late apoptosis. Control group exhibited consistently green live cells with regular and large nucleus morphology, but the cells incubated with different extracts displayed orange, red and yellow stain (fig. 4). fgRE was found to be more effective towards cancer cells in comparison to other plant extracts, with its most intense action on MDA-MB-231 cells and this may be due to the sensitivity of cells in comparison to other (MCF7). Again the important point to consider here was that apoptosis induced by the ivPE was comparable to fgRE and fgSE.
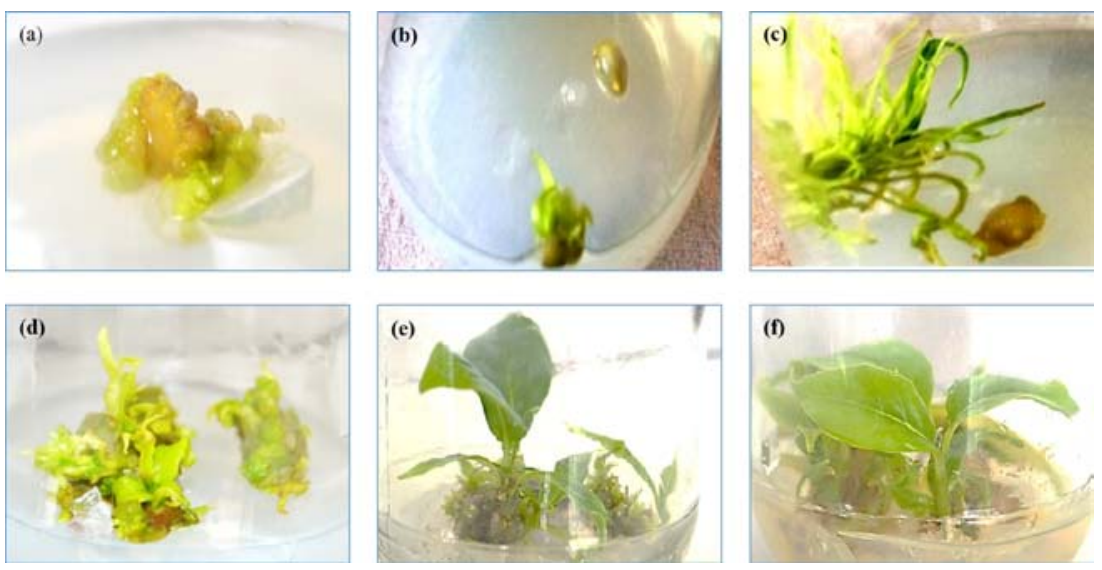

Fig. 1: In vitro shoot propagation from the somatic embryo of $N$. nimmoniana. a) Callus mass from field grown explant b) Somatic seed formed from selected somatic embryos from the callus mass c) Germinated somatic seed into micro shoots d) Cultured micro shoots to form in vitro grown plantlets after $2 \mathrm{w}$ of incubation e) and d) In vitro grown plantlets after two months of incubation in culture conditions
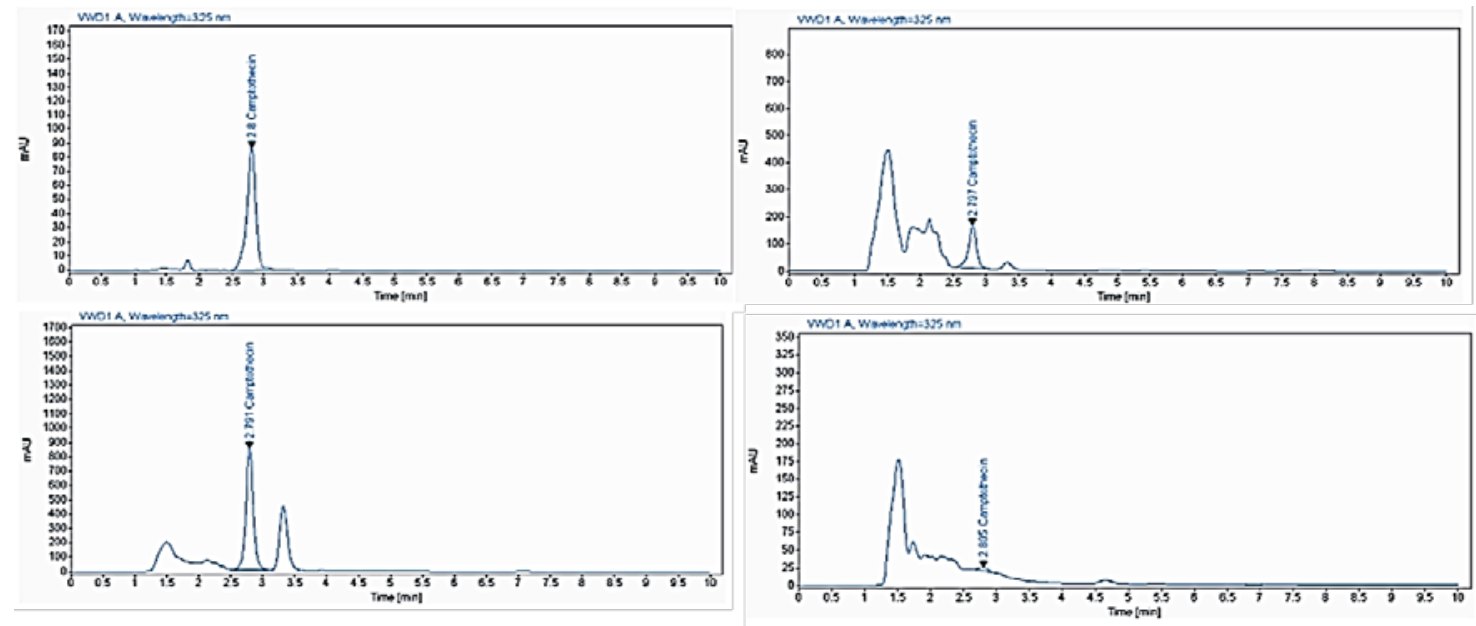

Fig. 2: Corresponding HPLC Graphs of; standard camptothecin (a), the field has grown shoots extract (b), the field has grown root extract (c) and recorded for in vitro plantlets extract (d) 


\section{Quantification of RNS}

In the past decade and so, it's turned into apparent that rise in the concentration of RNS facilitates neoplastic programmed cell death and constrains the cancer propagations [15]. Henceforth, we tend to measure $\mathrm{NO}$ level in extract treated cells and remarkably witnessed that fgRE had induced NO in both the cancer cell line to a greater extent. Whereas ivPE was not as much effective in inducing NO driven damage to cancer cells (fig. 5).

Quantification of ROS
Subsequently, we estimated the extract persuaded cell apoptotic trigger signalling responses via intra-cellular formation of ROS. ROS is well known to damage proteins, DNA and lipid membranes, and thereby disrupting the standard functional reliability of cells. $\mathrm{H}_{2} \mathrm{O}_{2}$ is the main constituent of the ROS and accountable for promoting apoptosis [17]. fgRE had generated the highest level of $\mathrm{H}_{2} \mathrm{O}_{2}$ in both cancer cell lines in contrast to other plant extracts, whereas, ivPE induced minimal $\mathrm{H}_{2} \mathrm{O}_{2}$ level (Fig.6). These results suggested that plant extract increased total cellular $\mathrm{H}_{2} \mathrm{O}_{2}$ generation triggering apoptosis in both cancer cell lines.
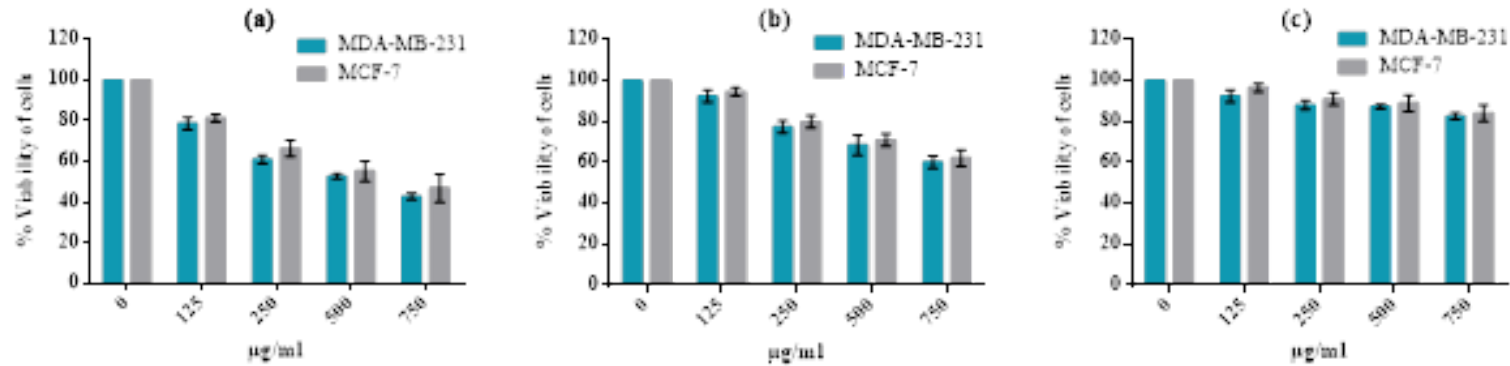

Fig. 3: Dose-dependent effects of field grown root extract (a), field has grown shoot extract (b) and in vitro plant extract (s) on MCF-7 and MDA-MB-231 cells respectively measured by MTT assay. Data are shown as mean \pm SEM of three separated experiments

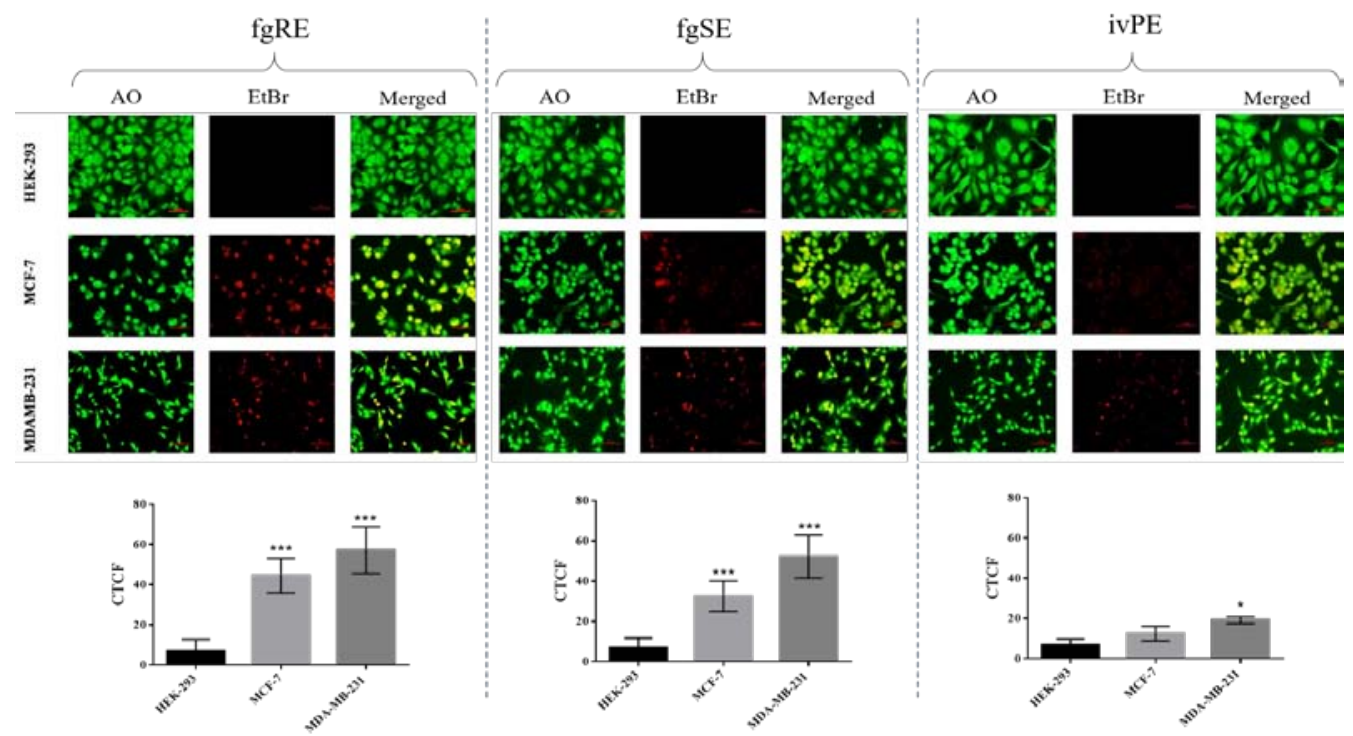

Fig. 4: AO and EtBr staining representing the effect of field grown root extract, field-grown shoot extract and in vitro plant extract on HEK293, MCF-7, and MDA-MB-231 cells respectively. The scale bar in images corresponds to $50 \mu \mathrm{M}(200 \mathrm{X})$. The graph represents the CTCF ratio for red fluorescence indicating dead PI-stained cells as result of cellular interaction with AGNPs at different time intervals
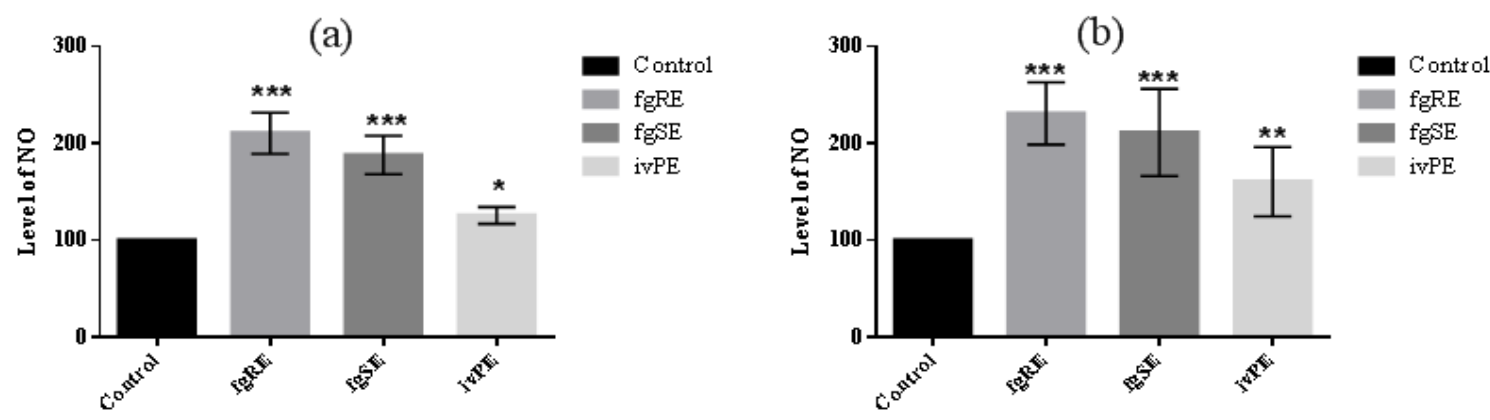

Fig. 5: Percentage of NO level as an effect of field grown root extract, field has grown shoot extract and in vitro plant extract on MCF-7 (a) and MDA-MB-231 cells (b. Bars represents mean $\pm \operatorname{SEM}(\mathrm{n}=3) . *$ indicates $\mathbf{p}<0.05, * *$ indicates $\mathbf{p}<0.01$ and $* * *$ indicates $\mathbf{p}<0.001$ when comparison made with control. Likewise, ${ }^{\text {indicates }} \mathbf{p}<0.05$, \#\# indicates $\mathbf{p}<0.01$ and $\# \# \#$ indicates p<0.001 in comparison to combination group for both cell lines (MCF-7 and MDA-MB-231) 
(a)

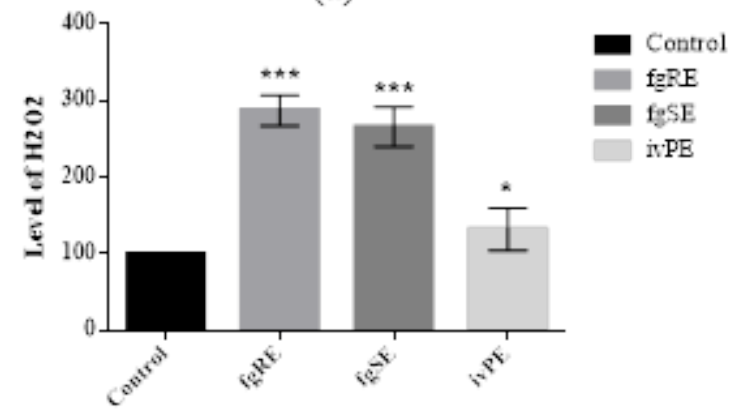

(b)

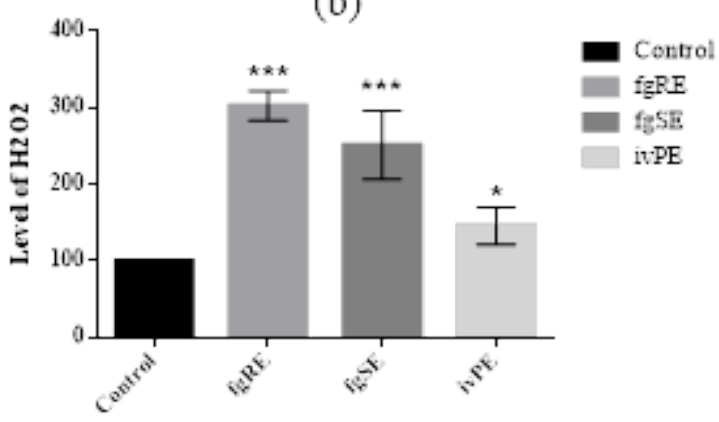

Fig. 6: Percentage of $\mathrm{H}_{2} \mathrm{O}_{2}$ level as an effect of field grown root extract, field grown shoot extract and in vitro plant extract on MCF-7 (a) and MDA-MB-231 cells $(b)$. Bars represents mean $\pm \operatorname{SEM}(n=3) .{ }^{*}$ indicates $p<0.05$, ${ }^{* *}$ indicates $p<0.01$ and $* * *$ indicates $p<0.001$ when comparison made with control. Likewise, \# indicates $p<0.05$, \#\# indicates $\mathbf{p}<0.01$ and \#\#\#indicates $\mathbf{p}<0.001$ in comparison to combination group for both cell lines (MCF-7 and MDA-MB-231)

Table 1: The IC 50 values determined from the plot of percent cytotoxicity on the cancerous cell line (MCF-7 and MDA-MB-231)

\begin{tabular}{lll}
\hline IC50 & MCF-7 & MDA-MB-231 \\
fgRE & 704.464 & 652.28 \\
fgSE & 1079.53 & 1025 \\
ivPE & 2997.75 & 2850.75 \\
\hline
\end{tabular}

\section{CONCLUSION}

A comparative examination of the extract acquired from field grown plant (5years old plants) and in vitro grown plant ( 2 mo old) have been investigated on breast cancer cells (MCF-7 and MDA-MB-231) and normal epithelial cells (HEK-293 cells). HPLC results revealed the presence of camptothecin in all extracts and the order was; fgRE $>$ fgSE $>$ ivPL. Field grown root extract outperformed the other extract by representing maximum efficiency towards cancer cells and this may be attributive to the highest concentration of CAM. ROS and RNS \% was also maximum in fgRE. Although the ivPL was not as efficient as fgRE, but it was intreating to note that ivPL was obtained from only 2 mo grown plant and fgRE was procured from $5 \mathrm{y}$ old plant. Overall on a broader spectrum, the root extract was more efficient towards tumour cells, in contrast, to shooting and in vitro plantlets. There are tremendous scope in near future for the researcher to improve the active compound biosynthesis acumination in in vitro shoots of $N$. nimmoniana by elicitor or enrichment studies. These in vitro shoots are comparatively very young and prompt alternative source to the field grown tissue for treating cancers and conserving ecosystem for future use.

\section{ACKNOWLEDGEMENT}

The authors are grateful to the Department of Biotechnology and Bioinformatics, Jaypee University of Information Technology, Solan, for financial support and providing laboratory facilities.

\section{AUTHORS CONTRIBUTIONS}

Arun Sharma and Ankita Rajata performed all the experiments and carried out the statistical analysis. They wrote the primary draft of this paper, which was further thoroughly rechecked by Dr. Hemant Sood and Dr. Udayabanu M. They have put necessary efforts to improve the quality of work and manuscript. All the authors had reviewed the manuscript and will provide further comments on future communication.

\section{ABBREVIATION}

CAM: Camptothecin

HPLC: High-performance liquid chromatography

ROS: Reactive-oxygen species

RNS: Reactive-nitrogen species

fgRE: Field grown root extract
fgSE: Field grown shoot extract

ivPL: In vitro plantlets

\section{CONFLICT OF INTEREST}

Authors have no conflict of interest among themselves

\section{REFERENCES}

1. Ramesha BT, Amna T, Ravikanth G, Gunaga RP, Vasudeva R, Ganeshaiah KN, et al., Prospecting for camptothecines from Nothapodytes nimmoniana in the Western Ghats, South India: identification of high-yielding sources of camptothecin and new families of camptothecines. J Chromatog Sci 2008;46:362-8.

2. Shaanker UR, Ramesha BT, Ravikanth G, Gunaga R, Vasudeva R, Ganeshaiah KN. Chemical profiling of Nothapodytes nimmoniana for camptothecin, an important anticancer alkaloid: towards the development of a sustainable production system. Bioactive molecules and medicinal plants. SpringerVerlag, Berlin and Heidelberg; 2008. p. 197-213.

3. Dancey J, Eisenhauer E. Current perspectives on camptothecins in cancer treatment. Br J Cancer 1996;74:327-38.

4. Moertel CG, Schutt AJ, Reitemeier RJ, Hahn RG. Phase II study of camptothecin (NSC-100880) in the treatment of advanced gastrointestinal cancer. Cancer Chemother Rep 1972;56:95-101.

5. Manikandan M, Kannan K. Study on in vivo release and in vivo absorption of camptothecin-loaded polymeric nanoparticles: level a in vitro-in vivo correlation. Asian J Pharm Clin Res 2016;9:71-4.

6. Srivastava V, Negi AS, Kumar JK, Gupta MM, Khanuja SP. Plantbased anticancer molecules: a chemical and biological profile of some important leads. Bio Med Chem 2005;13:5892-908.

7. Yamazaki Y, Sudo H, Yamazaki M, Aimi N, Saito K. Camptothecin biosynthetic genes in hairy roots of Ophiorrhiza pumila: cloning, characterization and differential expression in tissues and by stress compounds. Plant Cell Physiol 2003;44:395-403.

8. Cragg GM, Newman DJ. Plants as a source of anti-cancer agents. J Ethnopharmacol 2005;100:72-9.

9. Haisel D, Hofman P, Vagner M, Lipavska H, Ticha I, Schafer C, et al. Ex vitro phenotype stability is affected by in vitro cultivation. Bio Plant 2001;44:321-4.

10. Rajta A, Popli D, Kotvi P, Sood H. Optimization of culture conditions for the production and germination of artificial seed in an important medicinal plant, nothapodytes nimmoniana. Int J Innov Res Sci Eng 2017;3:70-7. 
11. Murashige T, Skoog F. A revised medium for rapid growth and bioassays with tobacco tissue cultures. Physiol Plants 1962; 15:473-97.

12. Shanthi G, Saridha D, Mariappan V. Pharmacognostical studies on morinda tinctoria. Roxb. Int J Pharm Pharm Sci 2012;4:636-8.

13. Lokesh P, Balasubramanya S, Anuradha M. Cost effective quantification of camptothecin and a comparative study of its content in Nothapodytes foetida and Ophiorrhiza mungos sourced from selected geographical locations. Ori Pharma Experiment Med 2014;14:87-92.

14. Aswini A, Soundhari C. Production of camptothecin from endophytic fungi and characterization by high-performance liquid chromatography and anticancer activity against colon cancer cell line. Asian J Pharm Clin Res 2018;11:166-70.

15. Mokhtar MM, Shaban HM, Hegazy ME, Ali SS. Evaluating the potential cancer chemopreventive efficacy of two different solvent extracts of seriphidium herba-alba in vitro. Bull Facult Pharma Cairo University 2017;1:195-201

16. Sharma A, Mehta V, Parashar A, Malairaman U. Combinational effect of paclitaxel and clotrimazole on human breast cancer: proof for synergistic interaction. Synergy 2017;5:13-20.

17. Wiseman H, Halliwell B. Damage to DNA by reactive oxygen and nitrogen species: role in inflammatory disease and progression to cancer. Biochem J 1996;313:17-29. 